\title{
Perturbed development of the mouse lens by polyomavirus large $T$ antigen does not lead to tumor formation
}

\author{
Anne E. Griep, ${ }^{1}$ Toichiro Kuwabara, ${ }^{2}$ Eric J. Lee, ${ }^{1}$ and Heiner Westphal ${ }^{1}$

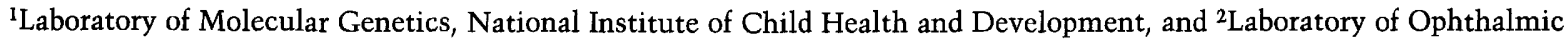 \\ Pathology, National Eye Institute, National Institutes of Health, Bethesda, Maryland 20892 USA
}

\begin{abstract}
To study how the oncogenic process may involve effects on differentiation, we overexpressed an immortalizing oncogene in a developing tissue in transgenic mice. By use of a gene fusion of the $\alpha \mathrm{A}$-crystallin promoter to the viral immortalizing oncogene, polyoma large $T$ antigen (PyLT), we created transgenic mice that express PyLT specifically in ocular lens. Expression of large $T$ antigen during embryonic development led to a perturbation in lens development, specifically, an interference with the normal program of fiber cell differentiation. This resulted in microphthalmia, which persisted throughout the life of the animal. Histological analysis revealed impairment of cell elongation, denucleation, and mitotic senescence in both primary and secondary fiber cell differentiation. Strikingly, there was no evidence for hyperplasia or for tumor development in vivo, unlike the consequences of many immortalizing oncogenes on tissues in other transgenic mice. In vitro, however, the developmentally perturbed cells derived from the transgenic lens showed high proliferative capacity. Our results suggest that a primary effect of aberrant expression of an immortalizing gene is an interference with normal tissue development; however, this interference may not necessarily induce proliferation or lead to tumor formation.
\end{abstract}

[Key Words: Immortalization; oncogene; lens; transgenic mouse; development]

Received February 16, 1989; revised version accepted April 25, 1989.

The challenge of understanding the process of tumorigenesis in vivo is being met by the use of the transgenic mouse model system. Evidence from recent transgenic studies indicates that certain oncogenes show a predisposition for affecting specific tissues (Adams et al. 1985; Leder et al. 1986; Rüther et al. 1987); that, whereas aberrant expression of one oncogene may lead to transformation, tumors form after a long latent period, suggesting the need for additional events (Stewart et al. 1984; Adams et al. 1985; Hanahan 1985; for review, see Palmiter and Brinster 1986; Cory and Adams 1988; Hanahan 1988); and that coexpression of oncogenes from different functional classes can act synergistically in tumor formation in vivo (Sinn et al. 1987; Andres et al. 1988). These classes are the immortalizing genes, exemplified by c-myc, polyoma large tumor $(\mathrm{T})$ antigen, and adenovirus E1A, and the transforming genes, including EJras and polyoma middle $\mathrm{T}$ antigen (Treisman et al. 1981; Rassoulzadegan et al. 1982, 1983; Land et al. 1983; Ruley 1983; for review, see Knudson 1986; Bishop 1987).

The function of an oncogene in the first class has been defined as immortalization on the basis of its ability to establish primary rodent cells in culture, allowing growth without crisis or senescence, and to allow an oncogene of the second class to transform these same cells if introduced concomitant with or subsequent to the immortalizing oncogene (Rassoulzadegan et al. 1982; Land et al. 1983; Ruley 1983). Recent work has suggested a role for the immortalizing oncogene in the regulation of growth and differentiation in vitro and in vivo. In in vitro studies, aberrant expression of c-myc and of polyomavirus large $\mathrm{T}$ antigen (PyLT) inhibited differentiation (Cherington et al. 1986; Coppola and Cole 1986; Griep and Westphal 1988), whereas depression of c-myc expression led to spontaneous differentiation (Griep and Westphal 1988; Holt et al. 1988). In transgenic mice, aberrant expression of c-myc during B-cell development led to proliferation of pre-B cells and to inhibition of pre-B-cell differentiation (Langdon et al. 1986), and aberrant c-myc expression in the mammary gland led to inhibition of alveolar development during lactation (Andres et al. 1988). Likewise, high expression of c-fos in bone led to increased osteoblastic activity in bone remodeling (Rüther et al. 1987), and expression of c-mos in the lens affected secondary fiber cell differentiation (Khillan et al. 1987). These in vivo results suggest that alterations in cellular differentiation result from aberrant expression of immortalizing oncogenes.

Later in life, the immunoglobulin-heavy-chain enhancer $(\mathrm{E} \mu)$-myc transgenic mice developed lymphomas (Adams et al. 1985), the whey acidic protein (WAP)-myc transgenic mice developed mammary adenocarcinomas (Schönenberger et al. 1988), and the c-fos transgenic mice developed chondrosarcomas or osteosarcomas 
(Wagner et al. 1988), tumors characteristic of the site of expression of the immortalizing oncogene. This suggests that aberrant expression of immortalizing oncogenes leads ultimately to tumor development. Strikingly, the c-mos transgenic mice did not develop lens tumors, although transgenic mice that express SV40 T antigen in the lens are known to develop lens tumors (Mahon et al. 1987). This difference in tumor promotion between mos and myc and fos could be explained by the lack of mos expression during the critical stages of lens development or, more interestingly, by the nature of the lens and its environment.

To explore these possibilities, we expressed the immortalizing oncogene PyLT specifically during the development of the lens. PyLT is the immortalizing oncogene of the murine papovavirus, polyoma (Rassoulzadegan et al. 1982), and is required for viral DNA replication (Francke and Eckhart 1973). Infection of neonatal mice with high titers of polyomavirus is known to result in a broad spectrum of tumor formation (Eddy 1969; Gross 1983). Despite the fact that polyoma is a murine virus, the effects of its large $T$ antigen in transgenic mice have not been studied extensively. For several reasons, the lens provides an attractive system in which to evaluate the consequences of aberrant oncogene expression on development and its relationship to tumorigenesis. The lens is composed of epithelial cells, which undergo a specific and well-characterized differentiation program (McAvoy 1978, 1980; for review, see Piatigorsky 1981). The $\alpha \mathrm{A}$-crystallin gene's transcriptional regulatory region has been shown to direct lens-specific transgene expression in transgenic mice (Overbeek et al. 1985). Furthermore, the activation of the $\alpha \mathrm{A}$-crystallin promoter in transgenic mice occurs early in the development of the lens (Overbeek et al. 1985; Mahon et al. 1987), similar to that of the endogenous gene (Zwann 1983). Thus, expression of an oncogene that is linked to this promoter occurs during the developmental stages of this specific tissue.

On the basis of histological and biochemical criteria, the transgenic mice that expressed the gene fusion of the $\alpha \mathrm{A}$-crystallin promoter to PyLT showed perturbed development of the lens, yet there was no evidence of tumor formation. This lack of tumor formation is unusual given that many immortalizing oncogenes have been shown to cause tumor formation in transgenic mice (Adams et al. 1985; Schönenberger et al. 1988; Wagner et al. 1988). Our results suggest that PyLT may be unique in its in vivo effects on tumor development. Alternatively, these results may indicate that a primary consequence of aberrant expression of immortalizing oncogenes is an interruption of normal cell differentiation; however, tumor formation requires additional events or factors that are not necessarily induced by the action of the immortalizing oncogene.

\section{Results}

\section{Generation of transgenic lineages}

The p $\alpha$ PyLT plasmid that was injected into fertilized mouse eggs is diagramed in Figure 1A. In this construc- tion, the coding sequence for the PyLT gene (Zhu et al. 1984) has been placed under the control of the murine $\alpha \mathrm{A}$-crystallin gene promoter (Chepelinsky et al. 1985), thus directing expression of large $\mathrm{T}$ antigen specifically to the lens of the transgenic mouse. Before microinjection, functional expression from poPyLT was tested in vitro by its ability to support replication of a plasmid that contained a polyoma origin of replication in lens cells. The origin-containing plasmids Py $\alpha \mathrm{CAT}$ and $\triangle \mathrm{Py} \alpha \mathrm{CAT}$, which contained the replication-competent origin and enhancer fragment of polyomavirus or the replication-defective origin fragment, respectively, are diagramed in Figure 1A. Transient replication assays were performed on primary lens cells and mouse fibroblast 3T3 cells that had been cotransfected with paPyLT and Py $\alpha \mathrm{CAT}$ or $\triangle \mathrm{Py} \alpha \mathrm{CAT}$. The plasmid p $\alpha$ PyLT supported replication of $\mathrm{Py} \alpha \mathrm{CAT}$ in lens cells but not in NIH-3T3 cells (Fig. 1B,C), which indicates that $\mathrm{p} \alpha \mathrm{PyLT}$ produced functional large $\mathrm{T}$ antigen specifically in the predicted cell type.

For microinjection, the $2.8-\mathrm{kb}$ SalI-XmaI fragment of p $\alpha$ PyLT, which contained the $\alpha \mathrm{A}$-crystallin promoter PyLT fusion gene, was injected into fertilized mouse eggs of the inbred strain FVB/N. Three of 24 pups were transgenic as determined by Southern blot analysis; one of the three founder mice apparently was mosaic as the transgene was not inherited by any progeny. The transgene was inherited in Mendelian fashion by progeny of the other two founder mice. Southern blot analysis determined that the copy numbers of the transgene per haploid genome were four for line $\alpha \mathrm{PyLTl}$ and one for line $\alpha$ PyLT2 (data not shown). The genotypes of progeny mice were indistinguishable from those of the founders, which suggested single-site integrations of the transgene (data not shown).

\section{Microphthalmia in $\alpha P y L T$ transgenic mice}

A dominant phenotypic trait of microphthalmia was observed in both founder mice, $\alpha$ PyLT1 and $\alpha$ PyLT2, and in all offspring that carried the transgene. The phenotype appeared consistent from generation to generation. However, in all other respects, the transgenic animals appeared normal. Homozygous transgenic mice exhibited the same phenotype and also showed no other noticeable abnormalities. At weaning age, the noticeably small cataractous lens of the transgenic mice made them clearly distinguishable from their normal littermates. The microphthalmia persisted throughout the life of the animal; the appearance of the lens was never normal.

The transgene was transferred into the $\mathrm{DBA} / 2 \mathrm{~J}$ and $\mathrm{C} 57 \mathrm{Bl} / 6 \mathrm{~N}$ backgrounds by the mating of an $\alpha$ PyLT1 mouse to a DBA/2J and a C57Bl/6N. Through four backcrossed generations to date, the microphthalmic phenotype has appeared as a dominant trait in all mice that carry the transgene. The presence of microphthalmia in two additional strains of mice indicates that this phenotype is not strain dependent.

Histological analysis of the lenses from the $\alpha$ PyLT1 transgenic mice was undertaken to assess the cause of 

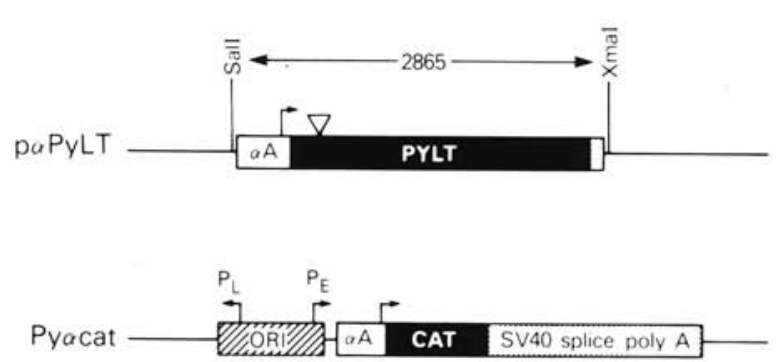

B.

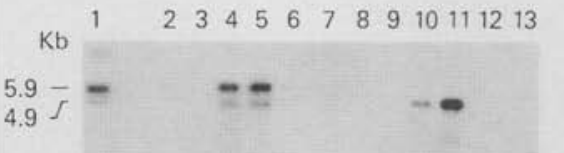

C.

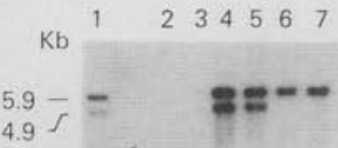

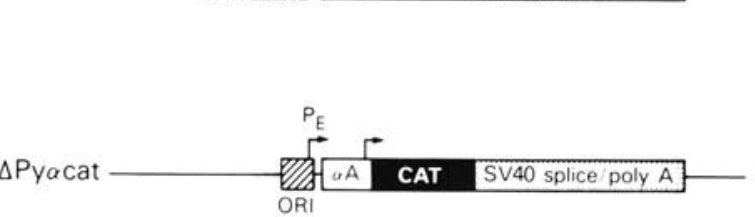

Figure 1. Construction and in vitro analysis of p $\alpha$ PyLT. $(A)$ The plasmids p $\alpha$ PyLT, Py $\alpha \mathrm{CAT}$, and $\triangle \mathrm{Py} \alpha \mathrm{CAT}$ were constructed as described in Methods. Solid blocks represent coding regions for polyoma large $T$ antigen or CAT; stippled blocks, polyadenylation signals; white blocks, $\alpha \mathrm{A}$-crystallin promoter; and hatched blocks, polyoma origin of replication. Also indicated are the transcriptional start sites in the $\alpha A$-crystallin promoter and the polyoma early $\left(\mathrm{P}_{\mathrm{E}}\right)$ and late $\left(\mathrm{P}_{\mathrm{L}}\right)$ promoters to show orientation of the polyoma origin sequences cloned into p $3364 \alpha \mathrm{CAT}$. (B) Southern blot analysis of replication competence of p $\alpha$ PyLT in NIH-3T3 cells. Hirt supernatant DNAs obtained after cotransfection of NIH-3T3 cells with the following plasmids were digested with BamHI and DpnI and analyzed by Southern blotting for intact polyoma origin sequences. Lanes refer to the plasmids which were cotransfected: (Lane 1) markers, $10 \mathrm{pg}$ of unit length linear, BamHI-digested Py $\alpha \mathrm{CAT}$ at $5.9 \mathrm{~kb}$, and $10 \mathrm{pg}$ of BamHI-digested pPyLTl at $4.9 \mathrm{~kb}$; (lanes 2 and 3 ) Py $\alpha$ CAT; (lanes 4 and 5) Py $\alpha$ CAT and pPyTL1; (lanes 6 and 7) Py $\alpha$ CAT and poPyLT; (lanes 8 and 9) $\Delta$ Py $\alpha$ CAT; (lanes 10 and 11 ) $\triangle \mathrm{Py} \alpha \mathrm{CAT}$ and $\mathrm{pPyTL} 1$; (lanes 12 and 13 ) $\triangle \mathrm{Py} \alpha \mathrm{CAT}$ and $\mathrm{p} \alpha \mathrm{PyLT}$. (C) Southern blot analysis of replication competence of p $\alpha \mathrm{PyLT}$ in primary lens epithelial cells. Analysis was performed as in $B$. Lanes refer to the plasmids that were cotransfected: (Lane 1) Marker, as in $B$; (lanes 2 and 3) PyaCAT; (lanes 4 and 5) Py $\alpha$ CAT and pPyLT1; (lanes 6 and 7) Py $\alpha$ CAT and p $\alpha$ PyLT. Low-molecular-weight hybridizing DNA represents unreplicated polyoma origin that contained DNA digested by DpnI. The plasmid pPyLT1, which contains the polyoma origin of replication as well as the large $\mathrm{T}$ antigen gene under the control of its homologous promoter, was used as a positive control for replication.

the microphthalmia. The normal adult lens is a large, dense, spherical structure. It is composed of an undifferentiated epithelium on the anterior surface and differentiated fiber cells that fill the remainder of the lens. The fiber cells are regularly shaped, elongated, postmitotic, and most are denucleated (Fig. 2A). By contrast, the structure of the adult $\alpha$ PyLT1 transgenic lens was distorted grossly (Fig. 2B). The transgenic lens was smaller than the normal age-matched lens, was collapsed at the equatorial region, and contained numerous large cysts. The cells were not properly elongated or denucleated. The posterior lens capsule appeared very thin and was found to be broken occasionally. Thus, the structural order of the $\alpha$ PyLT1 lens was distorted severely. The pathology of lenses from adult $\alpha$ PyLT2 mice exhibited the same abnormalities as did the $\alpha$ PyLT1 lens (Fig. 2C), which suggests that the cause of the microphthalmic phenotype in this lineage is the same as in the $\alpha$ PyLT1 lineage. The lens capsule of the $\alpha$ PyLT2 lens shown in Figure $2 \mathrm{C}$ was broken, which allowed for lens cells to escape the confines of the capsule and to reside in the ocular cavity. In lenses from homozygous $\alpha$ PyLT1 mice, the pathological features were similar (Fig. 2D). Details of the cellular pathology, including the persistence of nuclei, abnormal epithelium and capsule, as well as the presence of cysts and balloon cells, are visible more clearly at a higher magnification (Fig. 3A). The pathology of the $\alpha$ PyLT lenses suggests an impairment of normal lens development.

\section{Ontogeny of the $\alpha$ PyLT1 lens}

To determine whether the microphthalmia seen in the adult $\alpha$ PyLT lenses was a result of impaired development, $\alpha$ PyLT1 lenses were examined histologically at several stages in embryonic development. The normal developmental program of the lens proceeds in two stages. Primary fiber cell differentiation forms the nucleus of the lens and occurs around days 12 to 13.5 of embryogenesis. At this time, cells in the posterior portion of the lens vesicle become postmitotic, elongate to the anterior surface, and eventually lose their cell nuclei. Secondary fiber cell differentiation forms the cortex of the lens. It begins after the completion of primary fiber cell differentiation and continues throughout the life of the animal, although at a greatly reduced rate in the adult. Cells in the equatorial region of the lens undergo a terminal cell division cycle, elongate, eventually stretching from the posterior to the anterior surface, move toward the center of the lens, and lose their cell nuclei. Thus, both primary and secondary fiber cell differentiations are characterized by the onset of postmi- 

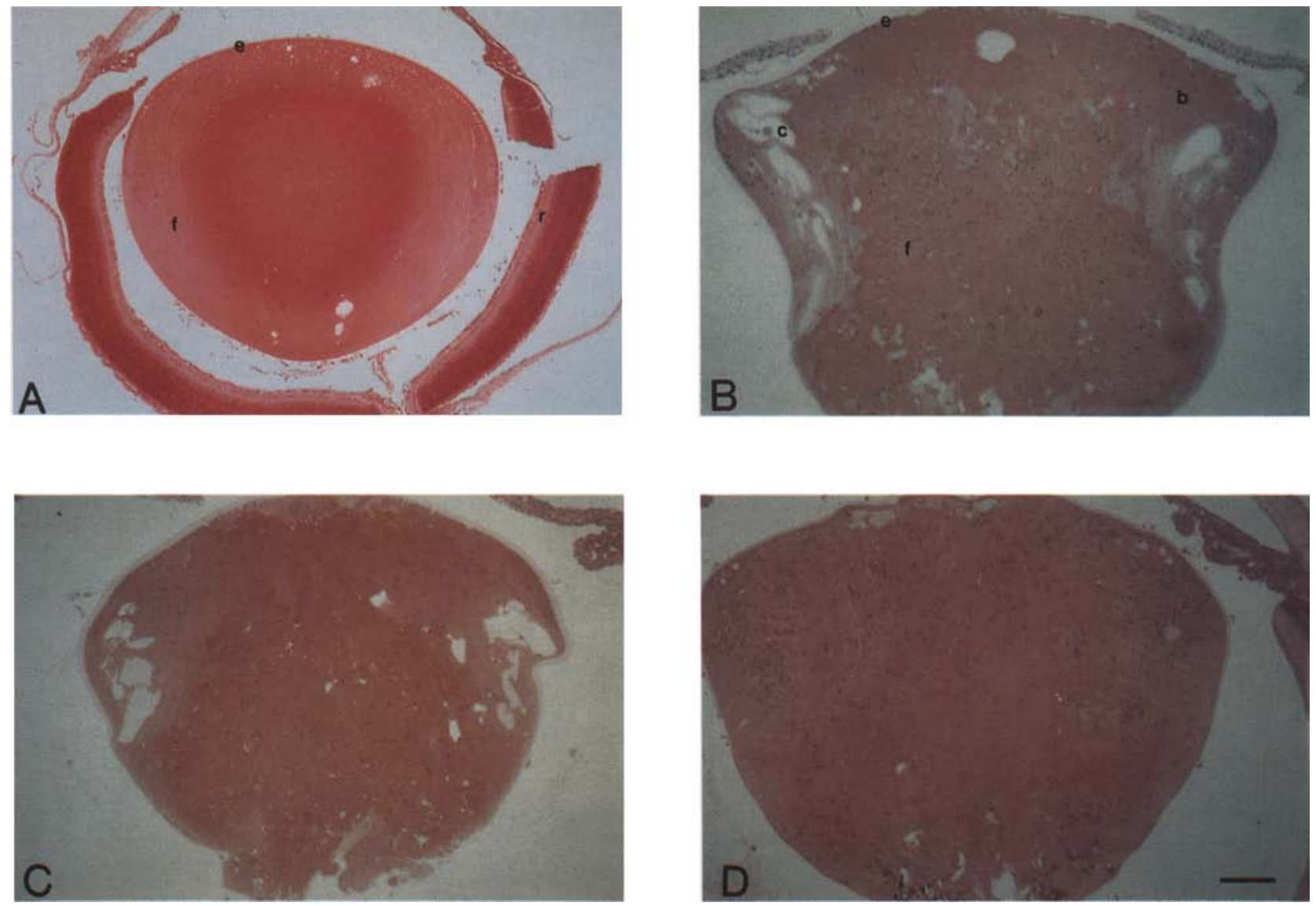

Figure 2. Histological analysis of lenses from adult FVB/N versus paPyLT transgenic mice. Representative hematoxylin and eosinstained sections of lenses are shown: $(A)$ a l-month-old FVB/N mouse; $(B)$ a 1-month-old $\alpha$ PyLT1 heterozygous transgenic mouse; $(C)$ a 1-month-old $\alpha$ PyLT2 heterozygous mouse; $(D)$ a 2-week-old $\alpha$ PyLT1 homozygous mouse. All sections are oriented with the posterior surface at the bottom and the anterior surface at the top. Bar, $18 \mu \mathrm{m}$ for $A$, and $11 \mu \mathrm{m}$ for $B, C$, and $D$. (e) Epithelial cells; (f) fiber cells; (r) retina; (c) cyst; (b) balloon cells. Note the large cysts in the bow region, the persistence of nuclei and nuclear material, balloon cells, and the overall shape and size of the lens compared to the normal lens.

totic terminal differentiation in which the cells become elongated and denucleated.

The developmental program of the normal lens is shown histologically in Figure 4, A-C. Figure 4A shows the lens at embryonic day 13.5 when primary fiber cell differentiation is completed and Figure 4, B and C, show two stages during secondary fiber cell differentiation, embryonic day 16.5 and neonate, respectively. Analysis of $\alpha$ PyLT1 lenses at these times revealed that both primary and secondary fiber cell differentiations were disrupted (Fig. 4D-F). The irregularly arranged, nucleated cells seen at embryonic day 13.5 (Fig. 4D) persisted at embryonic day 16.5 (Fig. 4E) and in the neonate (Fig. 4F). Likewise, elongation of fiber cells was impaired continually, which led to partially elongated or large balloon-like cells that failed to elongate, thereby resulting in numerous cysts. Finally, mitotic activity was present in the interior of the lens in normally postmitotic regions, and the denucleation process was retarded. These details of the cellular pathology are highlighted when the neonatal lens is viewed at a higher magnification (Fig. 3B). The fact that development is abnormal from the early stage of embryonic day 13.5 and persists, becoming more pronounced at each time point examined, indicates that the interference with fiber cell differentiation is progressive. These failures in differentiation leave the adult animal with the small opaque lens as seen in Figure 2; thus, the histological abnormalities of the embryonic $\alpha$ PyLT1 lenses indicate that the microphthalmia in these transgenic mice is the result of inhibition of both primary and secondary fiber cell differentiation.

\section{Expression of polyoma large $T$ antigen}

The abnormalities found in the $\alpha$ PyLT lenses were correlated with expression of PyLT protein by immunohistochemical and immunoblot analyses. Specific nuclear staining in a majority of the cells was seen in cryostat sections of newborn transgenic lenses that were immunostained by indirect immunofluorescence with monoclonal antibodies directed against PyLT (Fig. 5A,B). Staining was seen specifically in the lens and not in the surrounding ocular tissue. Similar immunostaining re- 

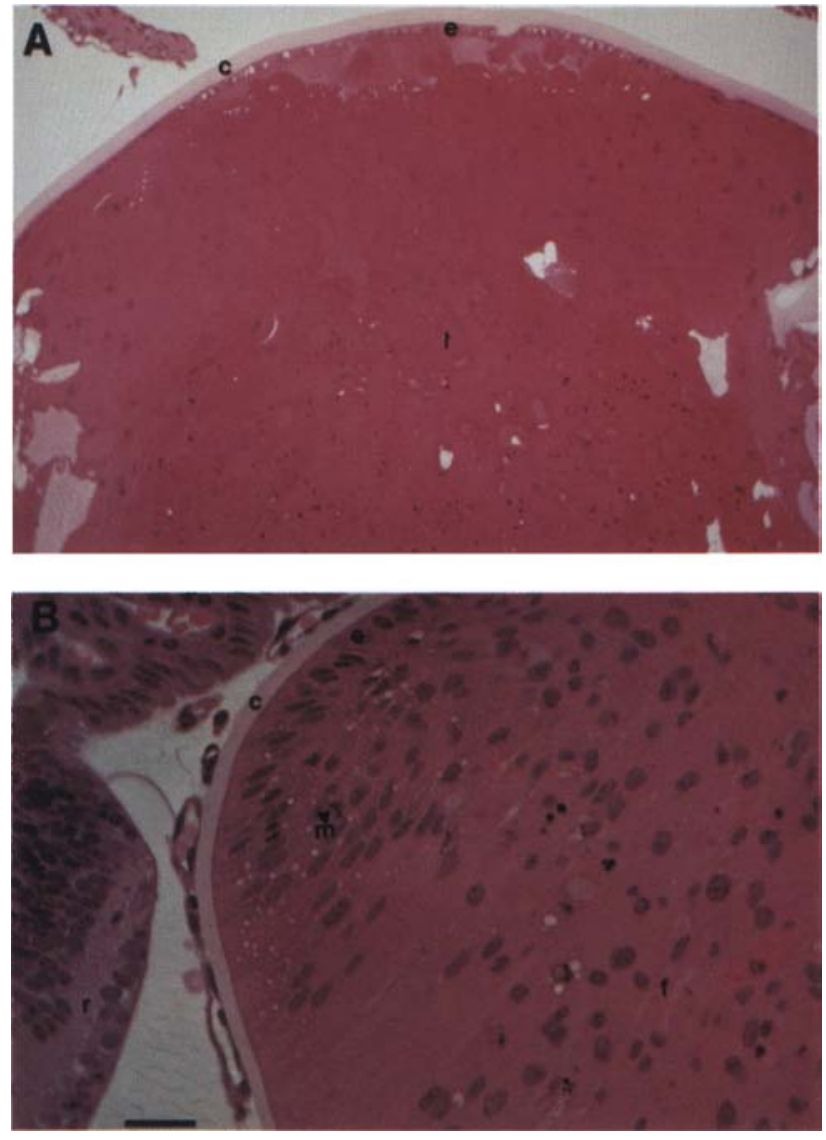

Figure 3. Histological analysis of $\alpha$ PyLT1 lenses at higher magnification. (A) Anterior portion of the section of the adult $\alpha$ PyLT2 lens shown in Fig. 2C. Bar, $8 \mu \mathrm{m}$. (B) Equatorial region of the neonatal $\alpha$ PyLT1 lens shown in Fig. 4F. Bar, $3 \mu \mathrm{m}$. (e) Epithelial cells; (f) fiber cells; (c) lens capsule; (m) mitosis.

sults were obtained with a polyclonal anti-large $\mathrm{T}$ antiserum as well (data not shown). PyLT, a protein of approximately $100 \mathrm{kD}$ (Ito et al. 1977), was found by immunoblot analysis by use of PyLT antibody specifically in protein lysates from adult transgenic eyes (Fig. 5C). PyLT was found only in the eyes of $\alpha$ PyLT1 mice but not in kidney, brain, or lung, which indicates the expected tissue specificity of expression (Fig. 5C). The level of protein found in lysates from eyes of homozygous $\alpha \mathrm{PyLTl}$ mice was greater than that in lysates from heterozygous eyes, indicative of a gene dosage effect, and the level of large $T$ antigen protein in lysates from eyes of the $\alpha$ PyLT2 mice was greater than that of the $\alpha$ PyLT1 line. Expression of PyLT at these three different levels resulted in similar lens pathology (Fig. 2). PyLT was detected in lysates from embryonic eyes at day 14.5 (Fig. 5C) and at days 13.5 and 16.5 (data not shown). PyLT was detected in lysates from lenses, but was not detected in lysates from residual eye tissue (data not shown). Expression of large $\mathrm{T}$ antigen at these early times is consistent with the expression patterns seen previously for other transgenes coupled to the $\alpha \mathrm{A}$-crystallin promoter (Overbeek et al. 1985; Mahon et al. 1987) and is similar to expression of the endogenous $\alpha \mathrm{A}$-crystallin gene (Zwann 1983). The fact that expression starts at this early time of 13.5 days in embryogenesis and continues into adult life supports the conclusion that the developmental abnormalities in the $\alpha$ PyLT lenses, which also start at this time, are the result of the action of PyLT.

\section{Expression of lens-specific crystallin genes}

To investigate differentiation of the transgenic lenses at a biochemical level, lenses and eyes were assayed for expression of the crystallin proteins by immunohistochemical and immunoblot analyses (data not shown). Indirect immunofluorescent staining of $\alpha-, \beta-$, and $\gamma$-crystallins in normal and transgenic neonatal lenses showed abnormal spatial distributions of crystallins in transgenic lenses. In some cells that normally make all three crystallin types, staining for $\alpha$ - or $\beta$-crystallins was not observed. Immunoblot analysis of normal and transgenic eyes and lenses suggested depressed levels of crystallins, particularly of $\gamma$ crystallins. Overall protein content of the transgenic lenses was lower than normal at the neonatal and adult stages. This supports the histological data that show a lack both of development and of hyperplasia in the transgenic lenses.

\section{Lack of tumor formation in old animals}

To determine whether malignant growth would occur spontaneously in transgenic animals that expressed PyLT, heterozygous and homozygous $\alpha$ PyLT1 animals of greater than 1 year of age were examined for the presence of eye tumors. No evidence of tumor formation was found on dissection, and no unusually high incidence of premature death was noticed. Histological examination of the eyes from heterozygous $\alpha$ PyLT1 animals at 13 and 16 months of age revealed no indication of hyperplasia, vascularization, or tumor formation. The lenses shown in Figure 6 are representative of two commonly found conditions, one in which the lens capsule is intact showing a small, poorly developed, sclerotic lens (Fig. 6A), and the other in which the lens capsule has ruptured showing lens cells residing in the ocular cavity to form a minilens attached to the original capsule (Fig. 6B). This contrasted with the histologies of the lenses from old control mice, which were similar to that of the normal lens shown in Figure 2A. The proliferative capacity of the $\alpha$ PyLT1 lens cells appeared low, and no evidence of tumor formation was found. Heterozygous $\alpha$ PyLT2 transgenic mice also have reached advanced age with no signs of a shortened lifespan or obvious pathology. Despite differences in the levels of large $\mathrm{T}$ antigen expression between these three examples of mice, the overall course of pathology, including the lack of tumor formation, was similar.

\section{Immortalization of lens cells by polyoma large T antigen}

To determine whether the $\alpha$ PyLTl lens cells were immortalized as defined by in vitro assays, cultured cell 

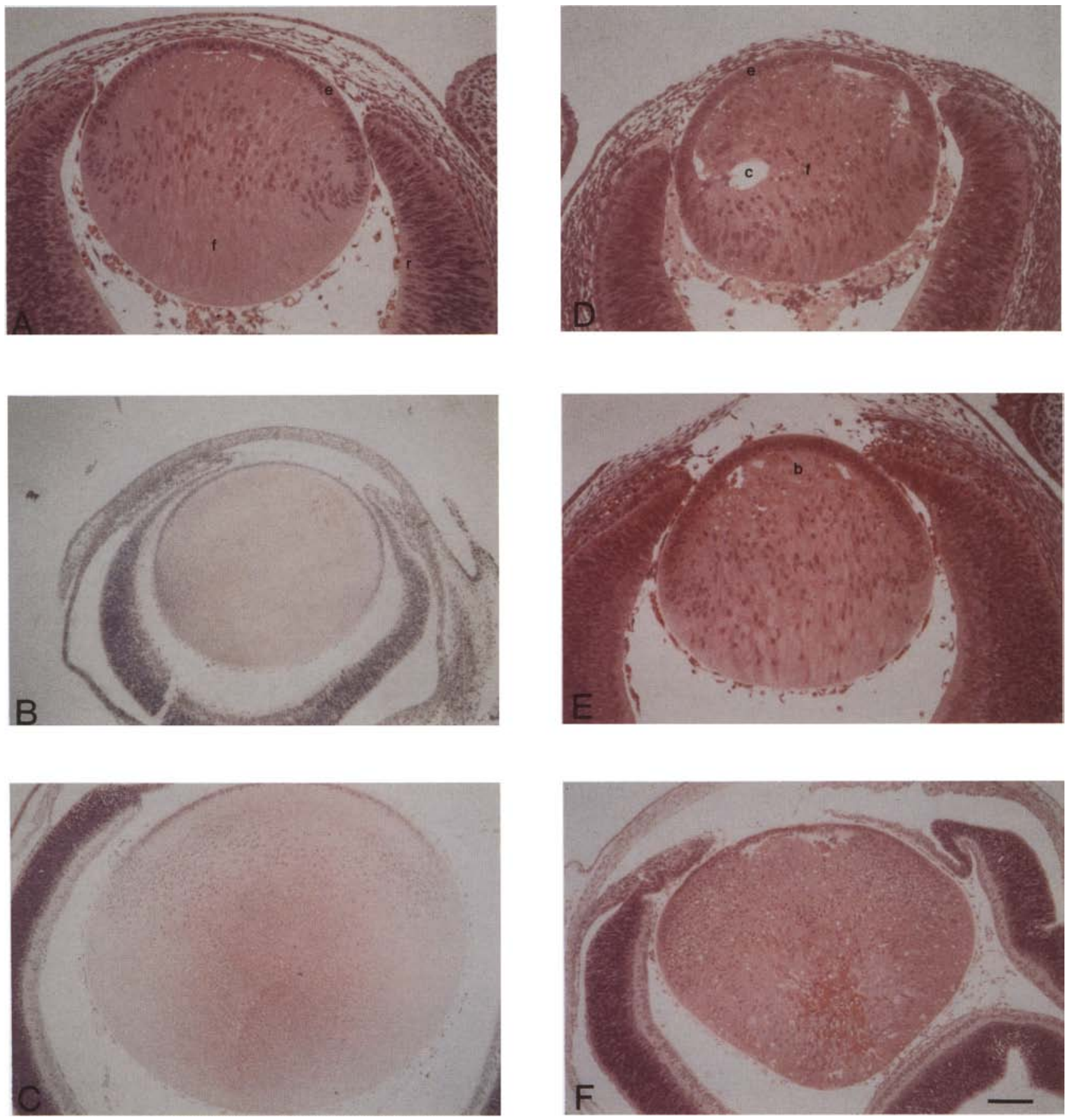

Figure 4. Histological analysis of lens development in FVB/N versus $\alpha P y L T 1$ transgenic mice. Representative hematoxylin and eosin-stained sections of lenses are shown: $(A)$ A 13.5-day FVB/N embryo; $(B)$ a 16.5-day FVB/N embryo; $(C)$ an FVB/N neonate; $(D)$ a 13.5-day $\alpha$ PyLT1 embryo; $(E)$ 16.5-day $\alpha$ PyLT1 embryo; $(F)$ an $\alpha$ PyLTl neonate. Note the abnormalities mentioned in Figs. 2 and 3. Bar, $5.5 \mu \mathrm{m}$ for $A, D$, and $E$, and $11 \mu \mathrm{m}$ for $B, C$, and $F$. $(e)$ Epithelial cells; $(\mathrm{f})$ fiber cells; $(\mathrm{r})$ retina; (c) cyst; (b) balloon cells.

lines were established from normal and $\alpha$ PyLTl transgenic neonatal lenses. Cultures initiated from normal neonatal lenses, which at first contained a mixture of both undifferentiated, proliferating epithelial cells, and differentiated nonproliferating fiber cells, gave rise to a population of epithelial cells that proliferated in culture for approximately 5-6 weeks, or 20 generations, before senescence occurred. Differentiation of these normal lens cells was evident in the presence of fetal bovine serum, and rapid terminal differentiation occurred in the presence of high levels of insulin (data not shown). Both fetal bovine serum and insulin are known to induce lens cell differentiation in vitro (Phillpott and Coulombre 1965; Piatigorsky 1973). By contrast, cells established in culture from the neonatal transgenic lenses showed no initial burst of differentiation and seemed resistant to 


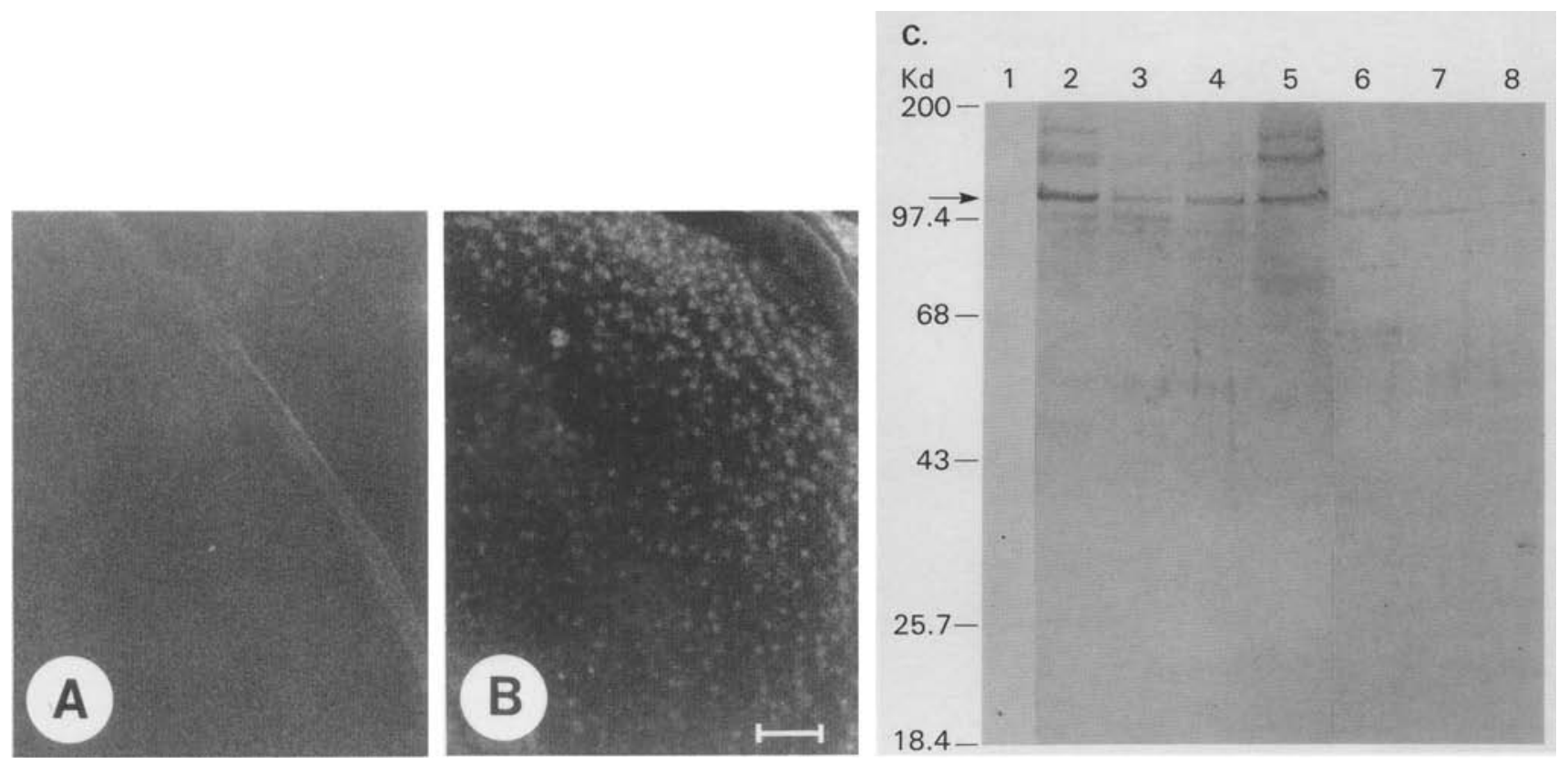

Figure 5. Analysis of PyLT expression in transgenic mice. $(A$ and $B)$ Analysis of PyLT expression by indirect immunoflourescence of cryostat sections from $\mathrm{FVB} / \mathrm{N}$ and $\alpha$ PyLT1 neonates. Representative sections of the equatorial region of the lens are shown immunostained with the Pb820 rat anti-polyoma large $\mathrm{T}$ antigen monoclonal antibody from: $(A) \mathrm{An}$ FVB/N neonate, and $(B)$ an $\alpha$ PyLT1 neonate. Bar, $5.5 \mu \mathrm{m} .(C)$ Analysis of PyLT antigen expression by immonoblotting. For analysis, $100 \mu \mathrm{g}$ of soluble RIPA lysates from the indicated tissues was electrophoresed on $7.5 \%$ SDS-polyacrylamide gels, electrotransferred, and immunoblotted with the hamster anti-polyoma large $\mathrm{T}$ antigen antibody. (Lanes 1-5) Lysates are from eyes. (Lane 1) 1-month-old FVB/N mice; (lane 2) 1-month-old $\alpha$ PyLT2 heterozygous mice; (lane 3) 1-month-old $\alpha$ PyLT1 heterozygous mice; (lane 4) 1-month-old $\alpha$ PyLT1 homozygous mice; (lane 5 ) 14.5-day $\alpha$ PyLTl embryos. (Lanes 6-8) Lysates are from tissues of 1-month-old $\alpha$ PyLT1 mice: (lane 6) kidney; (lane 7) brain; (lane 8) lung. Protein molecular mass standards are indicated (left). Arrow indicates PyLT. The band observed in kidney, brain, and lung also was observed in control $\mathrm{FVB} / \mathrm{N}$ tissues including eye (data not shown).

the differentiation-inducing factors in fetal bovine serum and to high levels of insulin. The large $T$ antigen lens cells appeared to proliferate indefinitely in vitro as they had not senesced after 6 months or 80 generations. The majority of these cells contained PyLT protein, as determined by indirect immunofluoresence (data not shown). In addition, the expression of the lenscell-specific proteins, the $\alpha$-crystallins, as detected by indirect immunofluorescence, confirmed the lenticular origin of these established cell lines. The characteristics of these cells, which were derived from the transgenic lenses, indicated that these cells were immortalized by in vitro definitions. The ability of these $\alpha$ PyLT1 cells to proliferate indefinitely in vitro, however, contrasted with their nonproliferative behavior in vivo. This difference could reflect the permissivity of the tissue culture environment in which passage permits for repeated outgrowth of subconfluent cultures, or it could reflect the nonpermissive environment of the lens cells within the eye (see Discussion section).

\section{Discussion}

\section{Impaired differentiation}

In this study we have shown that lens-specific expression of an immortalizing oncogene, PyLT, led to a mi- crophthalmic condition in which the development of the lens was perturbed. The lens pathology, indicative of impaired fiber cell differentiation, included failures in mitotic senescence, denucleation, and cell elongation (Figs. 2, 3, and 4). Biochemical analyses that demonstrated the presence of large $\mathrm{T}$ antigen in the lenses at least by embryonic day 14.5 (Fig. 5) suggested strongly that the abnormalities were a consequence of large-Tantigen action. Abnormal spatial and quantitative expression of the crystallin proteins and reduced protein content provided biochemical evidence for interrupted differentiation (data not shown). Thus, the data from our study suggest that PyLT inhibits lens cell differentiation. These findings are consistent with the fact that aberrant expression of other immortalizing oncogenes affects differentiation (Langdon et al. 1986; Rüther et al. 1987; Andres et al. 1988).

On the basis of our study, it is plausible that large $T$ antigen prevents recognition of differentiation-inducing signals which normally influence fiber cell differentiation. In vitro, normal lens cell differentiation can be induced by fetal bovine serum (Phillpott and Coulombre 1965), insulin (Piatigorsky 1973), or PDGF (Brewitt and Clark 1988), a situation similar to normal fiber cell differentiation in vivo in which factors secreted from the optic vesicle and retina such as lentropin, which is found in vitreous humor, influence differentiation 

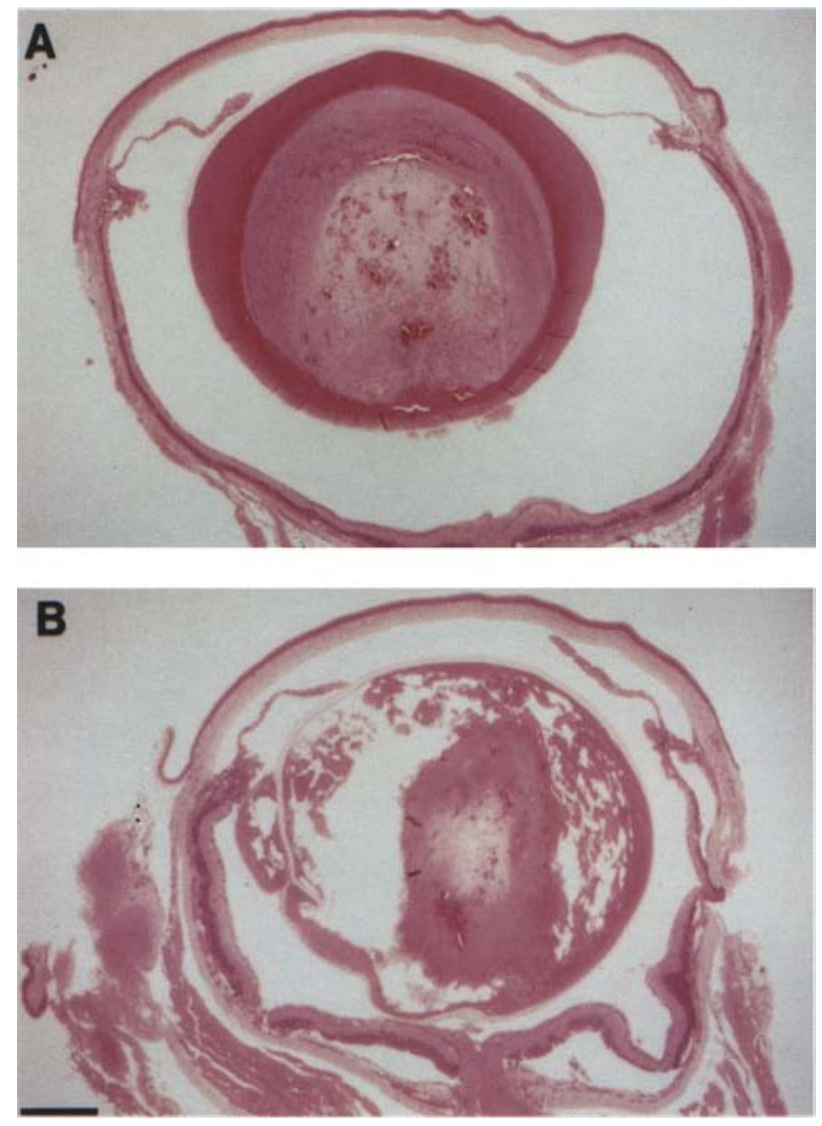

Figure 6. Histological analysis of lenses from old $\alpha$ PyLTl mice. $\langle A\rangle$ Section of the lens from a 16-month-old $\alpha$ PyLT1 mouse. Note the sclerosis in the lenticular nucleus, the poorly differentiated cortex, and the intact lens capsule. $|B|$ Section of the lens from a 13-month-old $\alpha$ PyLTl mouse. Note the large cystic space, in which partially differentiated cells of the cortex and the small sclerotic lenticular nucleus are floating, and the extracapsular cystic lens body outside the original capsule. Bar, $27 \mu \mathrm{m}$.

(Beebe et al. 1980). In vitro, cells derived from the aPyLT1 lens were resistant to the differentiation-promoting activity of fetal bovine serum and high doses of insulin (data not shown), recapitulating their behavior in vivo. Consistent with this hypothesis is the fact that the overexpression of PyLT in preadipocyte cells inhibits differentiation induced by adipogenic factors (Cherington et al. 1986). Also, expression of the immortalizing proto-oncogene $c-m y c$ is regulated during fiber cell differentiation (Nath et al. 1987). Collectively, these data suggest a possible link between differentiation inducers and regulators of cell growth, such as immortalizing oncogenes, during lens development. It is conceivable that the continuous action of PyLT in the lens supercedes the normal control circuit of differentiation inducers and gene products that regulate cell growth versus differentiation.

Our results showing that PyLT expression inhibits differentiation in the lens differ from those described previously for an alternative study on large $T$ antigen transgenic mice. Results from Bautch et al. (1987) indi- cated that expression of large $\mathrm{T}$ antigen had no effect on differentiation. The absence of developmental pathology in these transgenic mice may be the result of the very weak activity of the normal polyomavirus early promoter during embryonic development (Krippl et al. 1988).

\section{Lack of hyperplasia and tumor formation}

An interesting and important observation from this study is that tumor formation did not occur in the lenses of $\alpha$ PyLT transgenic mice. This is consistent with the view offered by the multistage transformation model that immortalizing oncogenes are not sufficient for transformation. Also consistent with this concept, tumors did form in the lens when both an immortalizing and transforming function were imposed by SV40 large T antigen (Mahon et al. 1987), yet our observations differ from those made with other transgenic mouse lines that harbored immortalizing oncogenes in which tumor formation was cited. Lymphoma, which was clonal, developed with variable latency in transgenic lines that harbored myc under the control of the immunogloublin heavy-chain enhancer, E $\mu$ (Adams et al. 1985), which suggested that secondary events were required. Similarly, tumor formation occurred in other transgenic mice with aberrant expression of c-myc (Stewart et al. 1984; Schönenberger et al. 1988) or c-fos (Wagner et al. 1988). The possibility that PyLT is not capable of eliciting tumors in transgenic mice can be discounted because an example of this has been observed (V. Bautch, pers. comm.). Conceivably, the lack of tumor formation in the large $T$ lens may be the result of a subthreshold level of large $\mathrm{T}$ antigen in the lens; however, because lowlevel polyoma large $T$ antigen expression was sufficient to cause tumorigenesis in another case, and aberrant immortalizing oncogene expression frequently causes tumorigenesis in transgenic mice, this explanation seems unlikely. Additionally, transgenic mouse lines that express another immortalizing oncogene (c-mos) in the lens, did not develop tumors (Khillan et al. 1987). It is more likely that the lack of tumor formation in the $\alpha$ PyLT mice reflects the cell type and cell environment in which the large $T$ antigen interacts. This interpretation also is consistent with the refractory nature of the lens to tumors that occur naturally (Sachs and Larsen 1948).

The tumorigenic progression observed in specific lines of transgenic mice that harbored immortalizing oncogenes commonly was preceded not only by disturbances in cell differentiation but also by increased proliferation of these cell populations (Langdon et al. 1986; Rüther et al. 1987, Andres et al. 1988). Further, given that the potential for spontaneous mutations is at least in part proportional to the frequency of DNA synthesis and repair events, this proliferative capacity of the cells is likely to be a determinative factor in tumor formation. Thus, in the case of the $\mathrm{E} \mu$-myc transgenic mice, myc expression resulted in the impaired differentiation and consequent expansion of the already substantial pre-B-cell popula- 
tion (Langdon et al. 1986), providing a large pool of proliferating cells in which mutations could occur. Similar proliferative disorders were noted in WAP-myc (Andres et al. 1988) and fos (Rüther et al. 1987) transgenic mice. Furthermore, the inherently unstable genome of the developing B cell may also contribute to the occurrence of a second event mutations in the $\mathrm{E} \mu$-myc transgenic mice (Langdon et al. 1986). By contrast, although the primary action of the immortalizing oncogene, PyLT, on the lenses of the PyLT mice was an inhibition of differentiation, proliferation was not induced (Figs. 2, 4, and 6). Thus, although inhibition of differentiation may favor proliferation over differentiation, the action of the oncogene does not appear to be sufficient to induce proliferation of the lens cells in vivo. Consequently, the likelihood for secondary events that lead to tumor progression potentially is restricted in the $\alpha$ PyLT mouse lens.

The lack of hyperplasia in the $\alpha$ PyLT lenses may be the result of unique characteristics of lens cells or to the environment of the lens. In the normal lens, the population of proliferating cells is very small as most cells are terminally differentiated. The growth potential of the lens is restricted because it is bound physically by the lens capsule. The lens is also avascular, which may be unfavorable for proliferation (Sachs and Larsen 1948). The $\alpha$ PyLT lens cells in vivo showed some abnormal proliferative capacity evidenced by mitoses in unusual regions of the lens (Fig. 3B). However, even in cases where the lens capsule had broken and lens cells had moved outside the capsule into the ocular cavity, high rates of proliferation did not occur (Figs. 2C and 6B). This lack of proliferation did not appear to be a property of the cells because, once removed from the in vivo environment of the lens to an in vitro environment, $\alpha$ PyLT lens cells proliferated extensively, whereas normal lens cells did not. This suggests that PyLT had conferred on the cell the ability to proliferate indefinitely when in a supportive environment. In conclusion, it is likely that the restriction of proliferation is imposed by the environment of the lens.

Evidence from our study may provide further support for the premise that proliferation is necessary for tumor formation. It has been suggested that development of a hyperplastic state usually does not arise as an immediate consequence of activated oncogene activity but requires further alterations in gene expression or genetic structure in the cell (Langdon et al. 1986; Hanahan 1988; Teitelman et al. 1988). The results of our study suggest that, in addition to the immediate action of the immortalizing oncogene on inhibiting differentiation, the cell type and environment may likely be among the determinative factors in promoting the development of a hyperplastic state. Indeed, the refractory nature of the $\alpha$ PyLT lens to the development of tumors suggests that these mice could be important to study further requirements for proliferation and second events in tumorigenesis. In this environment, the controlled introduction of growth factors or complementing transforming oncogenes, such as polyoma middle $\mathrm{T}$ antigen, may be useful to define the molecular mechanisms involved in tumor formation. The results presented in this study also emphasize the importance of immortalizing functions on tissue development in vivo. Comparison of the pathology of the lens in the $\alpha$ PyLT mice to cataracts in mouse (Gelatt and Das 1984), guinea pig, and man (T. Kuwabara, unpubl.) may provide some insight to the molecular events responsible for these pathological conditions.

\section{Methods}

Plasmid constructions and in vitro assays

The 412-bp BglII-BamHI $\alpha \mathrm{A}$-crystallin promoter fragment (Chepelinsky et al. 1985) was cloned into the BamHI site in pUC18. The 2.4-kb XhoI-BamHI PyLT cDNA fragment from the plasmid pspLT5 (Zhu et al. 1984) was cloned downstream of the $\alpha \mathrm{A}$-crystallin promoter at the BamHI site after blunt-end repair and BamHI-linker ligation of the $\mathrm{XhoI}$ end of the PyLT fragment to create the plasmid p $\alpha$ PyLT. The transcriptional start site is within the $\alpha \mathrm{A}$-crystallin promoter, whereas the translational initiation site and polyadenylation signal are within the PyLT fragment. The plasmid Py $\alpha$ CAT was created by insertion of the 800 -bp blunt-ended BamHI polyomavinus origin of replication and enhancer/promoter fragment [nucleotide numbers 4632-164 on the polyomavirus map (Soeda et al. $1980)]$ of pBgOri3 into the NdeI site in the $\alpha$ A-crystallin-CAT plasmid p $\alpha 364 \alpha$ CAT (Chepelinsky et al. 1985). The plasmid $\triangle \mathrm{Py} \alpha \mathrm{CAT}$ was created by the insertion of the blunt-ended 190 bp PvuII-BamHI polyomavirus origin and early promoter fragment (nucleotide numbers 5262-164 on the polyomavinus map) from the pBgOri3 into the NdeI site of p $\alpha 364 \alpha C A T$. The biological activity of paPyLT was tested in vitro in primary lens epithelial cells derived from 1-month-old FVB/N mice and in mouse fibroblast NIH-3T3 cells by cotransfection of equivalent amounts of $\mathrm{p} \alpha \mathrm{PyLT}$ or pPyLT1 (Rassoulzadegan et al. 1982) with Py $\alpha \mathrm{CAT}$ or $\triangle \mathrm{Py} \alpha \mathrm{CAT}$ by use of the calcium phosphate precipitation method (Graham and Vander Eb 1973). The episomal and chromosomal DNAs were isolated by the Hirt procedure (Hirt 1967) $48 \mathrm{hr}$ after transfection. Replicated DNA was identified by Southern blot analysis (Southern 1975) of material digested with BamHI and DpnI (Peden et al. 1980) with a ${ }^{32}$ P-labeled nick-translated (Rigby et al. 1977) 800 -bp polyoma origin fragment as probe. The plasmids pPyLT1 and pBgOri3 were obtained from F. Kern (New York University) and pspLT5 from C. Basilico (New York University).

\section{Microinjection and generation of transgenic lineages}

For microinjection, an XmaI-SalI 2.8-kb fragment from $\mathrm{p} \alpha$ PyLT was isolated by agarose gel electrophoresis and electroelution, and was purified by Elutip chromatography (Schleicher and Schuell, Keene, New Hampshire). The purified fragment DNA was injected into the male pronucleus of fertilized onecell embryos from $\mathrm{FVB} / \mathrm{N} \times \mathrm{FVB} / \mathrm{N}$ crosses as described previously (Overbeek et al. 1985; Hogan et al. 1986). The presence of the transgene was assayed by Southern blot analysis of genomic DNA from tail biopsies of 3-week-old mice with an internal fragment of large $\mathrm{T}$ antigen, the XhoI-KpnI 1.6-kb fragment from pspLT5, as probe.

\section{Histological analysis of the eye}

The eyes or heads (of embryos and newborns) were fixed in $4 \%$ glutaraldehyde in phosphate buffer $(\mathrm{pH} \mathrm{7.2)}$ for $30 \mathrm{~min}$ at room temperature, transferred to $10 \%$ formalin, and fixed for $24 \mathrm{hr}$. 
Vertically oriented central pieces were dissected, dehydrated in increasing concentrations of ethanol, and embedded in glycol methacrylate. From these specimens, $2-\mu \mathrm{m}$ sections were cut and stained with hematoxylin and eosin and examined by light microscopy. Embryos were staged by designating midday on the day of the vaginal plug as day 0.5 of development.

\section{Immunohistochemistry of neonatal lenses}

For analysis of PyLT expression, eyes of neonates from heterozygous $\alpha \mathrm{PyLT} 1$ transgenic by $\mathrm{FVB} / \mathrm{N}$ crosses were isolated and mounted in tissue-embedding medium (TissueTek-OCT) and flash frozen in isopentane that was cooled in liquid nitrogen. Sections $(6 \mu \mathrm{m})$ were cut on a cryostat at $-18^{\circ} \mathrm{C}$. Sections were fixed in acetone at $-20^{\circ} \mathrm{C}$ for $10 \mathrm{~min}$ and then washed in phosphate-buffered saline (PBS). Immunohistochemistry was performed as described previously (Mahon et al. 1987). Incubations with the anti-rat PyLT monoclonal antibody, Pb820, and with fluorescein isothiocyanate (FITC)-conjugated goat anti-rat IgG were for $1 \mathrm{hr}$ each at room temperature. The Pb820 monoclonal antibody was a gift from J. Bolen (National Cancer Institute, Bethesda, Maryland).

\section{Immunoblot analysis}

Eyes were isolated from transgenic and FVB/N mice of the ages indicated in the text and homogenized in a minimal volume of radioimmunoprecipitation (RIPA) buffer [50 mM Tris- $\mathrm{HCl}$ ( $\mathrm{pH}$ 7.4 |, $150 \mathrm{~mm} \mathrm{NaCl}, 1 \%$ Triton $\mathrm{X}-100,1 \%$ sodium deoxycholate, $0.1 \%$ SDS, $0.3 \mathrm{mg} / \mathrm{ml}$ PMSF]. Lenses were isolated from transgenic and FVB/N mice of the indicated ages by microdissection and were homogenized as for eyes. The protein content of soluble lysates was determined by the BCA protein-detection system (Pierce, Rockford, Illinois). Soluble lysates were electrophoresed on $7.5 \%$ SDS-Polyacrylamide gels, electrotransferred to nitrocellulose, and immunoblotted by use of the Vectastain ABC method (Vector Labs, Burlingame, California) The hamster anti-PyLT serum was a gift from A. Lewis (National Institute of Allergy and Infectious Diseases, Bethesda, Maryland).

\section{Establishment of primary lens cell cultures}

Lenses were isolated by microdissection from newborn FVB/N or $\alpha$ PyLT1 heterozygous mice. Lenses were treated briefly with trypsin solution and gentle trituration until the majority of the tissue was a single-cell suspension. After washing the cell suspension in medium [Dulbecco's modified Eagle's medium (DMEM), 10\% fetal bovine serum, $100 \mathrm{U} / \mathrm{ml}$ penicillin, 100 $\mu / \mathrm{ml}$ streptomycin] cells were plated in small drops of medium in tissue culture dishes for attachment and establishment of cultures. After expansion, the population was assayed for PyLT expression by immunostaining by use of the $\mathrm{Pb} 820$ rat antilarge $T$ antigen monoclonal antibody or hamster anti-large $T$ antigen antiserum mentioned previously. For growth in defined medium containing high insulin, cultures were passaged in DMEM growth medium then switched to the defined keratinocyte growth medium (KGM, Clonetics Corp., San Diego, California) containing $5 \mu \mathrm{g} / \mathrm{ml}$ of insulin for observation.

\section{Acknowledgments}

We thank Drs. Kathleen Mahon, Ernst-Martin Füchtbauer, and Paul Lambert for scientific discussions and assistance with the manuscript, Mike Sullivan for technical assistance, and Ms. Pat
McCubbin and Ms. Kathy Shoobridge for expert secretarial assistance.

\section{References}

Adams, J.M., A. W. Harris, C.A. Pinkert, L.M. Cocoran, W.S. Alexander, S. Cory, R.D. Palmiter, and R.L. Brinster. 1985. The c-myc oncogene driven by immunoglobulin enhancers induces lymphoid malignancy in transgenic mice. Nature 318: $533-538$.

Andres, A.C., M.A. VanderValk, C.-A. Schönenberger, F. Fluckiger, M. Lemeuri, P. Gerlinger, and B. Groner. 1988. Ha-ras and $\mathrm{c}-\mathrm{myc}$ oncogene expression interferes with morphological and functional differentiation of mammary epithelial cells in single and double transgenic mice. Genes Dev. 2: $1486-1495$.

Bautch, V.L., S. Toda, J.A. Hassell, and D. Hanahan. 1987. Endothelial cell tumors develop in transgenic mice carrying polyoma virus middle T oncogene. Cell 52: 529-538.

Beebe, D.C., D.E. Feagans, and A.J. Jebens. 1980. Lentropin: A factor in vitreous humor which promotes lens fiber cell differentiation. Proc. Natl. Acad. Sci. 77: 490-439.

Bishop, J.M. 1987. The molecular genetics of cancer. Science 235: 305-311.

Bloemendal, H. 1981. The lens proteins. In Molecular and cellular biology of the eye lens (ed. H. Bloemendal), pp. 1-47. John Wiley and Sons, New York.

Brewitt, B. and J.I. Clark. 1988. Growth and transparency in the lens and epithelial tissue stimulated by pulses of PDGF. Science 242: 777-779.

Chepelinsky, A.B., C.R. King, P.S. Zelenka, and J. Piatigorsky. 1985. Lens-specific expression of the chloramphenicol acetyltransferase gene promoted by the 5 '-flanking sequences of the murine $\alpha$-crystallin gene in explanted chicken lens epithelia. Proc. Natl. Acad. Sci. 82: 2334-2338.

Cherington, V., R. Morgan, R.M. Spiegelman, and J.M. Roberts. 1986. Recombinant retroviruses that transduce individual polyoma tumor antigens: Effects of growth and differentiation. Proc. Natl. Acad. Sci. 83: 4307-11.

Coppola, J.A. and M.D. Cole. 1986. Constitutive c-myc oncogene expression blocks mouse erythroleukaemia cell differentiation but not commitment. Nature 320: 760-765.

Cory, S. and J.M. Adams. 1988. Transgenic mice and oncogenesis. Annu. Rev. Immunol. 6: 25-48.

Eddy, B.E., 1969. Polyoma virus. In Virology monographs, vol. 7, pp. 1-114. Springer-Verlag, New York.

Francke, B. and W. Eckhart. 1973. Polyoma gene function required for viral DNA synthesis. Virology 55: 127-135.

Gelatt, K.N. and N.D. Das. 1984. Animal models for inherited cataracts: A review. Curr. Eye Res. 3: 765:778.

Graham, F.L. and A.J. Vander Eb. 1973. Transformation of rat cells by DNA of human adenovirus 5 . Virology 52: 456467.

Griep, A.E. and H. Westphal. 1988. Antisense myc sequences induce differentiation of F9 cells. Proc. Natl. Acad. Sci. 85: $6806-6810$.

Gross, L. 1983. The polyoma virus. In Oncogenic viruses, 3rd edition, vol. 2, pp. 737-828. Pergamon Press, Oxford.

Hanahan, D. 1985. Heritable formation of pancreatic B cell tumors in transgenic mice expressing recombinant insulin/ simian virus 40 oncogenes. Nature 315: 115-122.

. 1988. Dissecting multistep tumorigenesis in transgenic mice. Annu. Rev. Genet. 22: 479-519.

Hirt, B. 1967. Selective extraction of polyoma DNA from infected mouse cell cultures. J. Mol. Biol. 26: 365-369.

Hogan, B., F. Constantini, and E. Lacy. 1986. Manipulating the 
mouse embryo: A laboratory manual, Cold Spring Harbor Laboratory, Cold Spring Harbor, New York.

Holt, J.T., R.L. Redner, and A.W. Nienhuis. 1988. An oligomer complementary to c-myc mRNA inhibits proliferation of HL-60 promyelocytic cells and induces differentiation. Mol. Cell. Biol. 8: 963-973.

Ito, Y., N. Spurr, and R. Dulbecco. 1977. Characterization of polyoma virus T antigen. Proc. Natl. Acad. Sci. 74: 12591263.

Jamrich, M., K.A. Mahon, E.R. Gavis, and J.G. Gall. 1984. Histone RNA in amphibian oocytes visualized by in situ hybridization to methacrylate-embedded tissue sections. EMBO I. 3: 1939-1943.

Khillan, J.S., M.K. Oskarsson, F. Propst, T. Kuwabara, G.F. Vande Woude, and H. Westphal. 1987. Defects in lens fiber differentiation are linked to c-mos overexpression in transgenic mice. Genes Dev. 1: 1327-1335.

Knudson, A.F., Jr. 1986. Genetics of human cancer. Annu. Rev. Genet. 20: 231-251.

Krippl, B., A.E. Griep, K.A. Mahon, E. Bohnlein, P. Gruss, and H. Westphal. 1988. Expression and amplification in transgenic mice of a polyoma virus mutant regulatory region. Nucleic Acids Res. 16: 8963-8976.

Land, H., L.F. Parada, and R.A. Weinberg. 1983. Tumorigenic conversion of primary embryo fibroblasts requires at least two cooperating oncogenes. Nature 304: 596-602.

Langdon, W.Y., A.W. Harris, S. Cory, and J.M. Adams. 1986. The c-myc oncogene perturbs B lymphocyte development in E $\mu$-myc transgenic mice. Cell 47: 11-18.

Leder, A., P.K. Pattengale, A. Kuos, T.A. Stewart, and P. Leder. 1986. Consequences of widespread deregulation of the c-myc gene in transgenic mice: Multiple neoplasms and normal development. Cell 45: 485-495.

Mahon, K.A., A.B. Chepelinsky, J.S. Khillan, P.A. Overbeek, J. Piatigorsky, and H. Westphal. 1987. Oncogenesis of the lens in transgenic mice. Science 235: 1622-1628.

McAvoy, J.W. 1978. Cell division, cell elongation, and the coordination of crystallin gene expression during lens morphogenesis in the rat. J. Embryol. Exp. Morphol. 45: 271-281.

- 1980. Induction of the eye lens. Differentiation 17: $137-149$.

Nath, P., R. Getzenberg, D. Beebe, L. Pallansch, and P. Zelenka. 1987. c-myc mRNA is elevated as differentiating lens cells withdraw from the cell cycle. Exp. Cell Res. 169: 215-222.

Overbeek, P.A., A.B. Chepelinsky, J.S. Khillan, J. Piatigorsky, and H. Westphal. 1985. Lens-specific expression and developmental regulation of the bacterial chloramphenicol acetyl transferase gene driven by the murine $\alpha \mathrm{A}$-crystallin promoter in transgenic mice. Proc. Natl. Acad. Sci. 82: 78157819.

Palmiter, R.D. and R.L. Brinster. 1986. Germline transformation of mice. Annu. Rev. Genet. 20: 465-499.

Peden, K.W.C., J.M. Pipas, S. Pearson-White, and D. Nathans. 1980. Isolation of mutants of an animal vinus in bacteria. Science 209: 1392-1396.

Phillpott, G.W. and A.J. Coulombre. 1965. Lens development II. The differentiation of embryonic chick lens epithelial cells in vitro and in vivo. Exp. Cell Res. 38: 635-644.

Piatigorsky, J. 1973. Insulin initiation for lens fiber differentiation in culture: Elongation of embryonic lens epithelial cells. Dev. Biol. 30: 214-216.

. 1981. Lens differentiation in vertebrates. A review of cellular and molecular features. Differentiation 19: 134153.

Rassoulzadegan, M., A. Cowie, A. Carr, N. Glaichenhaus, R. Kamen, and F. Cuzin. 1982. The roles of individual polyoma virus early proteins in oncogenic transformation. Nature 300: $713-718$.

Rassoulzadegan, M., Z. Naghashfar, A. Cowie, A. Carr, M. Grisoni, R. Kamen, and F. Cuzin. 1983. Expression of the large $\mathrm{T}$ protein of polyoma virus promotes the establishment in culture of 'normal' rodent fibroblast cell lines. Proc. Natl. Acad. Sci. 80: 4354-4358.

Rigby, P.W., M. Diekmann, C. Rhodes, and P. Berg. 1977. Labeling deoxyribonucleic acid to high specific activity in vitro by nick-translation with DNA polmerase I. $/$. Mol. Biol. 113: 237-251.

Ruley, H.E. 1983. Adenovirus early region $1 \mathrm{~A}$ enables viral and cellular transforming genes to transform primary cells in culture. Nature 304: 602-607.

Rüther, R., C. Garber, K. Komitowski, R. Muller, and E.F. Wagner. 1987. Deregulated c-fos expression interferes with normal bone development in transgenic mice. Nature 325: 412-416.

Sachs, E., and R.L. Larsen. 1948. Cancer and the lens. Am. I. Ophthalomol. 31: 561-578.

Schönenberger, C.A., A.C. Andres, B. Groner, M. VanderValk, M. LeMeur, and P. Gerlinger. 1988. Targeted c-myc gene expression in mammary glands of transgenic mice induces mammary tumors with constitutive mild protein gene transcription. EMBO \%. 7: 169-175.

Sinn, W., W. Muller, A. Pattengale, I. Tepler, R. Wallace, and P. Leder. 1987. Coexpression of MMTV/v-Ha-ras and MMTV/ c-myc genes in transgenic mice: Synergistic action of oncogenes in vivo. Cell 49: 465-475.

Soeda, E., J.R. Arrand, N. Smolar, J.E. Walsh, and B.E. Griffin. 1980. Coding potential and regulatory signals of the polyoma virus genome. Nature 283: 445-453.

Southern, E.M. 1975. Detection of specific sequences among DNA fragments separated by gel electrophoresis. I. Mol. Biol. 98: 503-517.

Stewart, T.A., P.K. Pattengale, and P. Leder. 1984. Spontaneous mammary adenocarcinomas in transgenic mice that carry and express MTV/myc fusion genes. Cell 38: 627-637.

Teitelman, G., S. Albert, and D. Hanahan. 1988. Proliferation, senescence, and neoplastic progression of $\beta$ cells in hyperplastic pancreatic islets. Cell 52: 97-105.

Treisman, R., U. Novak, J. Favaloro, and R. Kamen. 1981. Transformation of rat cells by an altered polyoma virus genome expressing only middle- $\mathrm{T}$ protein. Nature 292: $595-600$.

Wagner, E.F., R.L. Williams, and R. Rüther. 1988. c-fos and polyoma middle- $T$ oncogene expression in transgenic mice and embryonal stem cell chimeras. In Cell to cell signals in mammalian development (ed. S.W. deLacy). SpringerVerlag, Berlin. (in press.)

Zhu, Z., G.M. Veldman, A. Cowie, A. Carr, B. Schaffhausen, and R. Kamen. 1984. Construction and functional characterization of polyomavirus genomes that separately encode three early proteins. I. Virol. 51: $170-180$.

Zwann, J. 1983. The appearance of $\alpha$-crystallin in relation to cell cycle phase in the embryonic mouse lens. Dev. Biol. 96: $173-181$. 


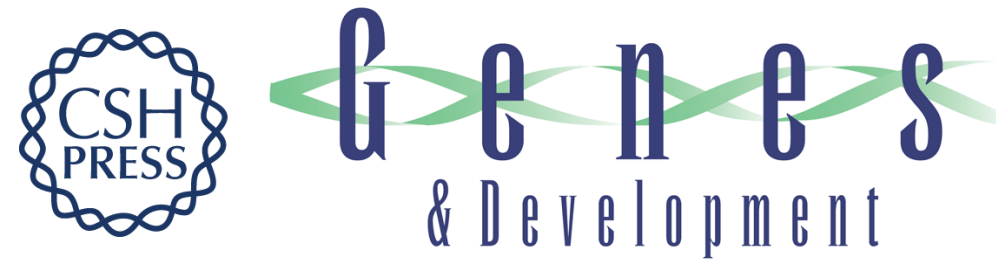

\section{Perturbed development of the mouse lens by polyomavirus large $T$ antigen does not lead to tumor formation.}

A E Griep, T Kuwabara, E J Lee, et al.

Genes Dev. 1989, 3:

Access the most recent version at doi:10.1101/gad.3.7.1075

References This article cites 49 articles, 13 of which can be accessed free at: http://genesdev.cshlp.org/content/3/7/1075.full.html\#ref-list-1

License

Email Alerting

Service

Receive free email alerts when new articles cite this article - sign up in the box at the top right corner of the article or click here.

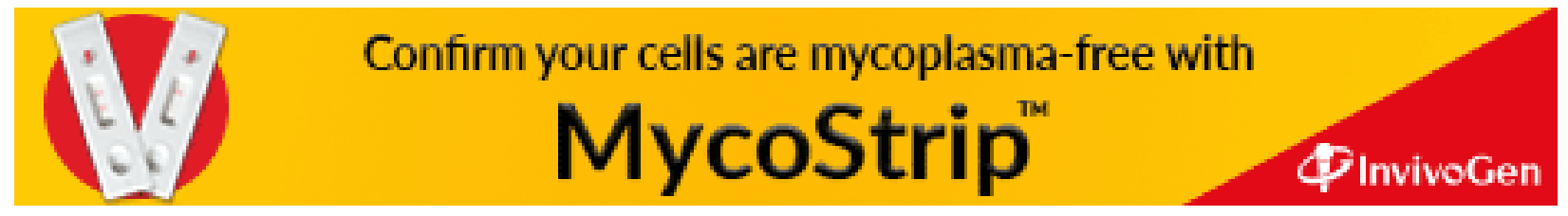

